\title{
RAPIDITY GAPS AND PRODUCTION OF MINIJETS IN HIGH-ENERGY HADRONIC COLLISIONS
}

\author{
G. Calucci, R. Ragazzon and D. Treleani \\ Dipartimento di Fisica Teorica dell'Università and INFN Sezione di Trieste \\ Trieste, I 34014 Italy
}

\begin{abstract}
High energy hadronic interactions can produce a final state characterized by minijets separated by a large gap in the rapidity distribution of the produced secondary particles. We discuss the process by keeping into account the possibility of having multiple parton collisions in the hadronic interaction. At Tevatron energy the correction to the single scattering term induced by the presence of multiparton interactions is large for transverse momenta smaller than $6 \mathrm{GeV}$.
\end{abstract}




\section{Introduction}

In the kinematical regime of semihard hadronic collisions the momentum transfer $q_{t}$ is large enough to apply perturbation theory but it is kept fixed with the c.m. energy, in such a way that the Regge limit $t / s \rightarrow 0$ is reached from the perturbative side. The main feature, which becomes more and more apparent when approaching the Regge limit, is the increasing complexity of the process. In fact at large $q_{t}$ several major aspects of the interaction are described by a single partonic collision represented with a Feynman diagram at the lowest order in the coupling constant. When moving towards the semihard regime the Regge limit is approached both by the hadronic and by the typical partonic collision. As a consequence the partonic process is not well represented any more by means of a tree level Feynman diagram. Partonic interaction in the Regge limit have been investigated extensively[1], [2]. An approach which has received a lot of attention is the BFKL Pomeron[2]: the partonic reaction is described by the exchange of a gluon ladder, with vacuum quantum numbers in the $t$-channel, which is constructed by neglecting the transverse momentum components when compared to the longitudinal ones. As a result of the simplified kinematics the steps of the ladder are ordered in rapidity and are build up with two basic elements:

a- the gauge independent non-local vertices, which sum up the dominant term, in the $t / s \rightarrow 0$ limit, of the diagrams with gluon emission from all near-by lines, and

b- the Reggeization of the $t$-channel gluons, which is introduced in order to keep into account the leading virtual corrections and which allows a solution to the infrared problem.

The iteration of the ladder in the $t$-channel, is expressed as an integral equation (Lipatov's equation) which can be solved explicitly. By considering the parton process represented with a cut BFKL Pomeron one may write explicitly the expression for the inclusive cross section to produce minijets, which are to be identified with final state partons with transverse momentum larger than the lower threshold of observability $q_{t}^{\text {min }}$. One may also consider the elastic parton interaction with BFKL Pomeron exchange. The elastic parton collision gives rise to a distinct signature in the final state generated by the parton process: two minijets are produced with no further particles in the rapidity interval between them. On the contrary in the typical inelastic parton collision, represented with a cut BFKL 
Pomeron, the gap is filled uniformly on the average by gluons. The dependence of the parton cross section on the rapidity gap is predicted by the perturbative calculation and it is related by unitarity to the dependence of the inelastic process on the width of the rapidity interval. The identification of these features in a semihard hadronic process would signal the underlying parton dynamics in a distinctive way[3]. A difficulty which has been pointed out is that the hadronic event which contains the semihard partonic interaction is going to fill the gap in most of the cases. The perturbative cross section with rapidity gap has therefore been multiplied by a survival probability factor[4]. The survival probability has been recently estimated by taking explicitly into account the underlying soft hadronic event and it turns out to be roughly constant as a function of the rapidity gap[5]. The underlying event would therefore provide only a rescaling factor to the cross section calculated perturbatively and the behaviour of the actual experimental cross section as a function of the rapidity gap would still be linked directly to the BFKL dynamics.

While testing the validity of the BFKL approach to semihard parton dynamics is presently one of the main topics in perturbative $\mathrm{QCD}$, the delicate point is to keep properly into account the structure of the whole hadronic interaction, whose effect may mask the BFKL dynamics[6]. In fact the BFKL regime requires $q_{t}^{\text {min }}$ small with respect to all longitudinal momenta and smaller values of $q_{t}^{\text {min }}$ correspond to larger values for the partonic cross section. In the BFKL regime unitarity corrections are therefore important. Indeed the closer is parton dynamics to the BFKL limit the stronger is the effect of unitarization[6].

In the present paper we discuss the effect of the unitarization of the semihard hadronic interaction on the cross section for minijet production with rapidity gaps in the distribution of final state secondaries. The unitarization of the hadronic semihard interaction induces multiple semihard partonic collisions in the inelastic event and the cross section to produce minijets with associated rapidity gap is therefore modified. To keep into account multiple BFKL Pomeron exchanges we assume the validity of the AGK cutting rules for semihard interactions. As a consequence the whole semihard hadronic process is represented as a probabilistic superposition of multiple BFKL Pomeron exchanges.

The paper is organized as follows: In the next paragraph the single parton interaction is discussed. The argument of the following section is multiple parton collisions; we describe the general formulation to the problem and we derive the 
expression for the cross section with rapidity gap in the simplest case of multiple parton interactions. A numerical example and some concluding remarks are the argument of the last section while a few possible generalizations are discussed in the appendix.

\section{Single scattering term}

By considering the cut BFKL Pomeron one may write the expression for the cross section where two gluons interact producing many gluons and two of them, the ones with largest rapidity (in absolute value) in the overall c.m. frame, are observed. If $y$ is the separation in rapidity and $k_{a}, k_{b}$ are the transverse momenta of the observed gluons, the inclusive cross section is expressed as

$$
\frac{d \hat{\sigma}_{L}}{d^{2} k_{a} d^{2} k_{b}}=\left[\frac{C_{A} \alpha_{s}}{k_{a}^{2}}\right] f\left(k_{a}, k_{b}, y\right)\left[\frac{C_{A} \alpha_{s}}{k_{b}^{2}}\right]
$$

where $C_{A}=N_{c}$ is the number of colors, $\alpha_{s}$ is the strong coupling constant and $f\left(k_{a}, k_{b}, y\right)$ is the inverse Laplace transform of the solution to Lipatov's equation. Actually:

$$
f\left(k_{a}, k_{b}, y\right)=\frac{1}{(2 \pi)^{2} k_{a} k_{b}} \sum_{\mathrm{n}=-\infty}^{+\infty} e^{i \mathrm{n} \phi} \int_{-\infty}^{+\infty} d \nu e^{\omega(\nu, \mathrm{n}) y} e^{i \nu \ln \left(k_{a}^{2} / k_{b}^{2}\right)}
$$

where $\phi$ is the azimuth angle between the observed gluons,

$$
\omega(\nu, \mathrm{n})=-2 \frac{\alpha_{s} N_{c}}{\pi} \Re\left[\psi\left(\frac{|\mathrm{n}|+1}{2}+i \nu\right)-\psi(1)\right]
$$

and

$$
\psi(z)=\frac{d \ln \Gamma(z)}{d z}
$$

is the Digamma function. The inclusive cross section for production of two minijets, as a result of cutting the exchange in the forward direction of a BFKL Pomeron, is obtained by folding Eq.(1) with the structure functions of the interacting hadrons $A$ and $B$ :

$$
\frac{d \sigma_{L}}{d x_{A} d x_{B} d^{2} k_{a} d^{2} k_{b}}=f_{e f f}\left(x_{A}, k_{a}^{2}\right) f_{e f f}\left(x_{B}, k_{b}^{2}\right) \frac{d \hat{\sigma}_{L}}{d^{2} k_{a} d^{2} k_{b}}
$$


where $f_{\text {eff }}$ is the effective structure function

$$
f_{e f f}(x)=G(x)+\frac{4}{9} \sum_{f}\left[Q_{f}(x)+\bar{Q}_{f}(x)\right]
$$

namely the gluon structure function plus $4 / 9$ of the quark and anti-quark structure functions with flavor $f$. In the BFKL dynamics one can relate the rapidity $y$ of the minijet which carries most of the momentum of the initial state parton (primary minijet) with the fractional momentum variable $x$ of the incoming parton. The relation is $x=k_{t} \mathrm{e}^{y} / \sqrt{s}$ for forward final state partons with transverse momentum $k_{t}$ and rapidity $y$ and $x=k_{t} \mathrm{e}^{-y} / \sqrt{s}$ for backward partons. Eq.(1) can be integrated on the transverse momenta down to the lower cut off $q_{t}^{\text {min }}$ :

$$
\hat{\sigma}_{L}(y)=\int_{q_{t}^{\text {min }}} \frac{d \hat{\sigma}_{L}}{d^{2} k_{a} d^{2} k_{b}} d^{2} k_{a} d^{2} k_{b}=\left(\frac{\alpha_{s} C_{A}}{\pi}\right)^{2} \frac{\pi^{3}}{2\left(q_{t}^{\text {min }}\right)^{2}} F_{L}(y)
$$

where

$$
F_{L}(y)=\int \frac{d \nu}{2 \pi} \frac{1}{\nu^{2}+1 / 4} e^{\omega(\nu) y}
$$

with $\omega(\nu) \equiv \omega(\nu, 0)$ as a consequence of the integration on $\phi . \hat{\sigma}_{L}$ is the inclusive cross section for minijet production in a parton process represented by a cut BFKL Pomeron. A different possibility which one may consider is to produce two minijets without cutting the BFKL Pomeron[7], namely by elastic scattering of two partons which exchange a BFKL Pomeron at momentum transfer larger than the lower cut off $q_{t}^{\text {min }}$. The corresponding integrated partonic cross section is expressed as[3]:

$$
\hat{\sigma}_{S}=\left(\frac{\alpha_{s} C_{A}}{\pi}\right)^{4} \frac{\pi^{5}}{4\left(q_{t}^{\text {min }}\right)^{2}} F_{S}(y)
$$

and $F_{S}(y)$ is the convolution of two BFKL propagators in the transverse momentum plane:

$$
F_{S}(y)=\frac{\left(q_{t}^{\text {min }}\right)^{2}}{16 \pi^{3}} \int_{q_{t}^{\text {min }}} d^{2} k\left|\int d^{2} q d^{2} q^{\prime} f^{k}\left(q, q^{\prime}, y\right)\right|^{2}
$$

$k$ is the overall momentum exchanged through the ladder and the label $S$ refers to the singlet exchange in the elastic partonic collision. Obviously one might consider also the possibility of having a octet exchange, the contribution to the cross section 
is however subleading at large rapidities[3]. The integrals on $q$ and $q^{\prime}$ in Eq.(8) can be performed when using the integral representation of $f^{k}\left(q, q^{\prime}, y\right)$ :

$$
\int d^{2} q d^{2} q^{\prime} f^{k}\left(q, q^{\prime}, y\right)=\frac{4}{k^{2}} \int d \nu \frac{\nu^{2}}{\left(\nu^{2}+1 / 4\right)^{2}} \exp [\omega(\nu) y]
$$

and one obtains the asymptotic behavior at large $y$

$$
\hat{\sigma}_{S}(y) \simeq\left(\frac{\alpha_{s} C_{A}}{\pi}\right)^{4} \frac{\pi^{5}}{4\left(q_{t}^{\min }\right)^{2}}\left(\pi \frac{e^{4 \ln 2 z}}{\left(\frac{7}{2} \zeta(3) \pi z\right)^{3 / 2}}\right)^{2}
$$

where $z=\alpha_{s} C_{A} y / \pi$. For comparison the asymptotic behavior of $\hat{\sigma}_{L}$ is:

$$
\hat{\sigma}_{L}(y) \simeq\left(\frac{\alpha_{s} C_{A}}{\pi}\right)^{2} \frac{\pi^{3}}{2\left(q_{t}^{\min }\right)^{2}}\left(\frac{e^{4 \ln 2 z}}{\left(\frac{7}{2} \zeta(3) \pi z\right)^{1 / 2}}\right)
$$

The 'elastic' partonic cross section can be identified with the 'diffractive' cut of the double BFKL Pomeron exchange contribution to the forward parton amplitude[8]. At the same order, in the number of exchanged BFKL Pomerons, one needs therefore to keep into account also the one BFKL Pomeron cut and two BFKL Pomeron cut contributions to the partonic process. In fact the relative rate of elastic and inelastic parton processes, as expressed in Eq.(12) and in Eq.(13), is not consistent at large rapidity intervals because of the too rapid rise of the elastic cross section. We do not try to solve here this unitarity problem. To have an indication on the boundaries of the kinematical regime where the problem needs to be faced we have taken the simplest attitude. In analogy to the $s$-channel unitarization of the soft Pomeron exchange we have included in the semihard partonic interaction the exchange of two BFKL Pomerons and we have used the AGK cutting rules[9] to obtain the inelastic contributions to the cross section. The semihard partonic cross section $\hat{\sigma}_{H}(y)$ is therefore expressed as

$$
\hat{\sigma}_{H}(y)=\hat{\sigma}_{S}(y)+\left(\hat{\sigma}_{L}(y)-4 \hat{\sigma}_{S}(y)\right)+2 \hat{\sigma}_{S}(y)
$$

where the single BFKL Pomeron exchange contributes with $\hat{\sigma}_{L}(y)$ and the contributions from the double BFKL Pomeron exchange, according with the AGK cutting rules, are: $\hat{\sigma}_{S}(y)$, the 'diffractive' contribution i.e. neither Pomeron is cut, $-4 \hat{\sigma}_{S}(y)$, the one Pomeron cut, and $+2 \hat{\sigma}_{S}(y)$, the two Pomeron cut. Eq.(14) allows one to define the kinematical region of applicability of the approach. Indeed the one BFKL cut Pomeron contribution to the cross section must be positive[10]: 


$$
\left(\hat{\sigma}_{L}(y)-4 \hat{\sigma}_{S}(y)\right)>0
$$

Eq.(15) fixes a limiting value to the rapidity interval $y$ as a function of $\alpha_{S}$, as an example for $\alpha_{s}=.18$ and $\alpha_{S}=.22$ one obtains about 14 and 10 rapidity units respectively. Strictly speaking the contribution expressed by Eq.(15) implies that the one-cut-Pomeron events and the two-cut-Pomeron events are distinguishable in term of their multiplicity. If the fluctuations in multiplicity prevents this identification we get the milder condition

$$
\left(\hat{\sigma}_{L}(y)-2 \hat{\sigma}_{S}(y)\right)>0
$$

which requires only the distinction between diffractive and non-diffractive events.

We therefore express the semihard cross section $\hat{\sigma}_{H}(y)$, corresponding to a single partonic interaction, as

$$
\hat{\sigma}_{H}(y)=\hat{\sigma}_{S}(y)+\left(\hat{\sigma}_{L}(y)-2 \hat{\sigma}_{S}(y)\right) \equiv \hat{\sigma}_{S}(y)+\hat{\sigma}_{P}(y)
$$

and $\hat{\sigma}_{P}(y)$ is the contribution from production of secondaries, both from one and two cut BFKL Pomerons.

\section{General Framework for Multiparton Interactions}

Given the scale $q_{t}^{\min }$ one may separate parton fluctuations with a lifetime long with respect to $q_{t}^{\min }$ and parton fluctuations with a lifetime short with respect to $q_{t}^{\text {min }}$. Parton fluctuations which have a long lifetime are of non-perturbative origin and can be associated to the initial state. Parton fluctuations with a short lifetime can be treated perturbatively and can be associated to the semihard interaction. In the simplest case, when the typical value of the rapidity interval $y$ in the partonic interaction is not too large, the semihard partonic interaction is described at the lowest order in the number of exchanged BFKL Pomerons. If $q_{t}^{\min }$ is relatively small one faces however a unitarity problem even if one is in a regime where the partonic interaction is well described within perturbation theory because of the large value of the integrated semihard hadronic cross section[11]: by integrating Eq.(5) with the cut off $q_{t}^{\min }$ one obtains a cross section which easily exceeds the value of the total cross section. On the other hand the partonic cross section, 
Eq.(16), is in comparison still rather small. The large value of the integrated inclusive cross section is therefore the consequence of the large flux of partons in the initial state, which gives rise to an average number of partonic collisions larger than one[12]. In the typical semihard hadronic process different partonic interactions are localized in the transverse plane in different regions, of size of order $\left\langle\hat{\sigma}_{H}\right\rangle$ inside the overlap volume of the two interacting hadrons, whose transverse size is of a few $\mathrm{fm}^{2}$. At Tevatron energy, considering partonic interactions with cross section $\hat{\sigma}_{H}$, as expressed in Eq.(16), and $q_{t}^{\text {min }}=6 \mathrm{GeV}$, the average distance in rapidity between produced minijets is about five units. The corresponding value of the partonic cross section is $\hat{\sigma}_{H}(\langle y\rangle) \simeq 8 \times 10^{-2} \mathrm{mb}$ and one may correspondingly estimate that in a central collision the average number of partonic interactions is $\simeq$ $2 \leftrightarrow 4$. These features characterize the kinematical regime which we are presently interested in: actually the 'elementary' partonic interaction is well described within the BFKL dynamics, in such a way that the bound in Eq.(15) is satisfied, and the inclusive hadronic minijet cross section is larger than the inelastic cross section, in such a way that the rate of multiple parton interactions is sizeable.

To discuss multiple parton collisions we follow the approach used in ref.[6] and $[13,14]$ : In the case of soft interactions multi-Reggeon exchanges are conveniently taken into account by making use of the AGK cutting rules[9]. Although no general proof of their validity is available in the case of semi-hard interactions, it has nevertheless been possible to show that the cutting rules hold for one of the components of the interaction which is leading in the large- $\hat{s}$ fixed- $\hat{t}$ limit[15]. If one assumes the validity of the cutting rules for semi-hard interactions, one is allowed to represent the semi-hard cross section $\sigma_{H}$ as a probabilistic distribution of multiple semi-hard parton collisions[14]. The most general expression for $\sigma_{H}$ requires however the introduction of the whole infinite set of multiparton distributions[16], which keep into account hadron fluctuations in the parton number. To that purpose we introduce the exclusive $k$-body parton distribution $W^{(k)}\left(u_{1} \ldots u_{k}\right)$, namely the probabilities to find a hadron in a fluctuation with $k$ partons with coordinates $u_{1} \ldots u_{k}, u_{i} \equiv\left(b_{i}, x_{i}\right)$ standing for the transverse partonic coordinate $\left(b_{i}\right)$ and longitudinal fractional momentum $\left(x_{i}\right)$. From the exclusive distributions and the auxiliary functions $J(u)$ one may construct the generating functional $\mathcal{Z}[J]$ :

$$
\mathcal{Z}[J]=\sum_{n} \frac{1}{n !} \int J\left(u_{1}\right) \ldots J\left(u_{n}\right) W_{n}\left(u_{1}, \ldots u_{n}\right) d u_{1} \ldots d u_{n}
$$


in such a way that the exclusive distributions are the coefficients of the expansion of the generating functional around $J=0$, while the inclusive distributions are the coefficients of the expansion around $J=1$. A very general expression for the semihard cross section, which is consistent with the cutting rules, is therefore:

$$
\begin{aligned}
\sigma_{H}=\int d^{2} \beta \sigma_{H}(\beta) \\
\sigma_{H}(\beta)=\int \sum_{n} \frac{1}{n !} \frac{\delta}{\delta J\left(u_{1}\right)} \cdots \frac{\delta}{\delta J\left(u_{n}\right)} \mathcal{Z}_{A}[J] \\
\times \sum_{m} \frac{1}{m !} \frac{\delta}{\delta I\left(u_{1}^{\prime}-\beta\right)} \cdots \frac{\delta}{\delta I\left(u_{m}^{\prime}-\beta\right)} \mathcal{Z}_{B}[I] \\
\times\left.\left\{1-\prod_{i=1}^{n} \prod_{j=1}^{m}\left[1-\hat{\sigma}_{H}\left(u_{i}, u_{j}^{\prime}\right)\right]\right\} \prod d u d u^{\prime}\right|_{J=I=0}
\end{aligned}
$$

Here the $\beta$ is the impact parameter between the two interacting hadrons and $\hat{\sigma}_{H}\left(u_{i}, u_{j}^{\prime}\right)$, represents the probability for the parton $i$ of the $A$-hadron to have a semihard interaction with the parton $j$ of the $B$-hadron. The semi-hard cross section is constructed by summing over all possible partonic configurations of the two interacting hadrons (the sums over $n$ and $m$ ) and, for each configuration with $n$ $A$-partons and $m B$-partons, summing over all possible multiple partonic interactions. This last sum is constructed by asking for the probability of no interaction between the two configurations (actually $\prod_{i=1}^{n} \prod_{j=1}^{m}\left[1-\hat{\sigma}_{i, j}\right]$ ). The difference from one of the probability of no interaction gives the sum over all semi-hard interactions. $\sigma_{H}(\beta)$ is then the probability to have at least one semihard parton interaction when the impact parameter in the hadronic collision is equal to $\beta$. The semi-hard cross section is obtained by integrating the probability $\sigma_{H}(\beta)$ on the impact parameter. Analogously, the elementary semi-hard cross section $\hat{\sigma}_{H}\left(x, x^{\prime}\right)$ is obtained by integrating the elementary interaction probability $\hat{\sigma}_{H}\left(u, u^{\prime}\right)$ on the relative transverse coordinate $\mathbf{b}-\mathbf{b}^{\prime}$.

In Eq.(18) $\sigma_{H}$ is constructed by summing all possible semihard two-body parton collisions. Multiple semi-hard parton collisions are of two distinct kinds, disconnected collisions and rescatterings. In a disconnected collisions different pairs of partons interact independently at different points in the transverse plane. In a rescattering a high energy parton interacts several times, with momentum exchange larger than $q_{t}^{\text {min }}$, with different target partons and all interactions are 
localized in the same region, approximately of size $\left(1 / q_{t}^{\min }\right)^{2}$, in the transverse plane. The most important contribution to the semi-hard cross section arises from the disconnected partonic collisions. In fact, at a given number of partonic collisions, the incoming parton flux is maximized in the configuration where all collisions are disconnected. It might therefore be meaningful to obtain a simpler expression for $\sigma_{H}$ by neglecting the rescattering processes in Eq.(18). To that purpose we expand the interaction probability ( the factor in curly brackets ) as sums and suppress all addenda containing repeated indices:

$$
\left\{1-\prod_{i, j}^{n, m}\left[1-\hat{\sigma}_{i j}\right]\right\} \Rightarrow \sum_{i j} \hat{\sigma}_{i j}-\frac{1}{2 !} \sum_{i j} \sum_{k \neq i, l \neq j} \hat{\sigma}_{i j} \hat{\sigma}_{k l}+\ldots
$$

Because of the symmetry of the derivative operators in Eq.(18) one can replace the expression in Eq.(19) with:

$$
n m \hat{\sigma}_{11}-\frac{1}{2 !} n(n-1) m(m-1) \hat{\sigma}_{11} \hat{\sigma}_{22}+\ldots
$$

in such a way that the sums over $n$ and $m$ in Eq.(18) can be performed explicitly. As a consequence the cross section at fixed impact parameter $\sigma_{H}(\beta)$ is expressed by the operatorial form:

$$
\sigma_{H}(\beta)=\left.\left[1-\exp \left(-\frac{\delta}{\delta J} \cdot \hat{\sigma} \cdot \frac{\delta}{\delta I}\right)\right] \mathcal{Z}_{A}[J+1] \mathcal{Z}_{B}[I+1]\right|_{J=I=0}
$$

where the dependence on the variables $u$ and $u^{\prime}$ is understood.

The expression of $\sigma_{H}(\beta)$, as given by Eq.(21), is still too complicated to be worked out, since all possible multi-parton correlations are implicitly present in $\mathcal{Z}$. The simplest possibility is to neglect all correlations in the multi-parton distributions. In this case one writes

$$
\mathcal{Z}[J+1]=\exp \int D(u) J(u) d u
$$

where $D(u)$ is the average number of partons. The cross section assumes therefore the eikonal form:

$$
\sigma_{H}=\int d^{2} \beta[1-\exp (-\Phi(\beta))]
$$

where 


$$
\Phi(\beta) \equiv \Phi_{S}(\beta)+\Phi_{P}(\beta) \equiv \int_{y_{m}}^{y_{M}} d y \int_{y}^{y_{M}} d y^{\prime}\left(\phi_{S}\left(\beta ; y, y^{\prime}\right)+\phi_{P}\left(\beta ; y, y^{\prime}\right)\right)
$$

with

$$
\phi_{S, P}\left(\beta ; y, y^{\prime}\right) \equiv \int d^{2} b D_{A}(b, x(y)) \hat{\sigma}_{S, P}\left(y^{\prime}-y\right) D_{B}\left(b-\beta, x^{\prime}\left(y^{\prime}\right)\right)
$$

and $y_{M}, y_{m}$ are the maximum and minimum rapidity values allowed by kinematics. The index $S$ in Eq.(24) refers to the elastic parton interaction, the singlet exchange in Eq.(16), and the index $P$ to the inelastic interaction, the one and two cut Pomeron contributions in Eq.(16). The different contributions from multiple semihard parton collisions to the cross section are explicit if one expands the exponential in Eq.(23) as follows:

$$
\begin{aligned}
\sigma_{H} & =\int d^{2} \beta \sum_{\nu=1}^{\infty} \frac{1}{\nu !} e^{-\Phi(\beta)} \\
& \times \int_{y_{1}<y_{1}^{\prime}} d y_{1} d y_{1}^{\prime}\left(\phi_{S}\left(\beta ; y_{1}, y_{1}^{\prime}\right)+\phi_{P}\left(\beta ; y_{1}, y_{1}^{\prime}\right)\right) \\
& \cdots \\
& \times \int_{y_{\nu}<y_{\nu}^{\prime}} d y_{\nu} d y_{\nu}^{\prime}\left(\phi_{S}\left(\beta ; y_{\nu}, y_{\nu}^{\prime}\right)+\phi_{P}\left(\beta ; y_{\nu}, y_{\nu}^{\prime}\right)\right)
\end{aligned}
$$

One is interested in the component of $\sigma_{H}$ which represents two minijets at rapidities $\bar{y}$ and $\bar{y}^{\prime}$, in the central rapidity region, with associated gap $\Delta y=\bar{y}^{\prime}-\bar{y}$ in the rapidity distribution of secondary produced gluons. To that purpose one needs to exclude in Eq.(26) both the 'elastic' terms, with final state minijets in the gap, and all the inelastic partonic interactions, generated with elementary probability $\phi_{P}$. In fact the cut BFKL Pomeron originates soft gluons distributed on the average uniformly in rapidity. Local fluctuations in the rapidity distribution of soft gluons, which could leave the gap empty also in the case of an inelastic elementary parton interaction, may be neglected if one considers a reasonably large rapidity gap. The cross section to observe two minijets at rapidities $\bar{y}$ and $\bar{y}^{\prime}$, with the gap $\Delta y=\bar{y}^{\prime}-\bar{y}$ in the rapidity distribution of secondaries, is therefore expressed as 


$$
\begin{aligned}
\frac{d \sigma_{H}(\Delta y)}{d \bar{y} d \bar{y}^{\prime}}=\int & d^{2} \beta\left[\sum_{\nu=1}^{\infty} \nu \phi_{S}\left(\beta ; \bar{y}, \bar{y}^{\prime}\right) \frac{\left[\Phi_{S}(\beta, \Delta y)\right]^{\nu-1}}{\nu !}\right. \\
+\sum_{\nu=2}^{\infty} \nu(\nu-1) \int_{y_{m}}^{\bar{y}} d y \phi_{S}\left(\beta ; y, \bar{y}^{\prime}\right) \int_{\bar{y}^{\prime}}^{y_{M}} d y^{\prime} \phi_{S}\left(\beta ; \bar{y}, y^{\prime}\right) & \left.\times \frac{\left[\Phi_{S}(\beta, \Delta y)\right]^{\nu-2}}{\nu !}\right] e^{-\Phi(\beta)}
\end{aligned}
$$

where

$$
\Phi_{S}(\beta, \Delta y) \equiv \int_{y_{m}}^{\bar{y}} d y \int_{\bar{y}^{\prime}}^{y_{M}} d y^{\prime} \phi_{S}\left(\beta ; y, y^{\prime}\right)
$$

After summing on $\nu$ one obtains

$$
\begin{array}{r}
\frac{d \sigma_{H}(\Delta y)}{d \bar{y} d \bar{y}^{\prime}}=\int d^{2} \beta\left[\phi_{S}\left(\beta ; \bar{y}, \bar{y}^{\prime}\right)+\int_{y_{m}}^{\bar{y}} d y \phi_{S}\left(\beta ; y, \bar{y}^{\prime}\right) \int_{\bar{y}^{\prime}}^{y_{M}} d y^{\prime} \phi_{S}\left(\beta ; \bar{y}, y^{\prime}\right)\right] \\
\quad \times \exp \left\{\Phi_{S}(\beta ; \Delta y)-\Phi_{S}(\beta)-\Phi_{P}(\beta)\right\}
\end{array}
$$

The two addenda in Eq.(29) are the single and double 'elastic' scattering terms. In the single scattering term both observed minijets are produced in the same elementary partonic interaction, in the double scattering term the two minijets are generated in different partonic collisions. Both terms are multiplied by the absorption factor $\exp \left\{-\left(\Phi_{S}(\beta)-\Phi_{S}(\beta ; \Delta y)\right)\right\}$ that removes the 'elastic' parton interactions which would fill the gap, actually those which produce minijets with rapidities $y$ and $y^{\prime}$ such that $\bar{y} \leq y$ or $y^{\prime} \leq \bar{y}^{\prime}$. At a fixed value of $\beta$ the cross section is multiplied by $\exp \left\{-\Phi_{P}(\beta)\right\}$ which is the probability of not having any inelastic partonic interaction in the process. One may recognize in Eq.(29) the semihard contribution to the survival probability factor $\left\langle S^{2}(\beta)\right\rangle$ of ref.[4]. Actually $\left.\exp \left\{-\Phi_{S}(\beta)-\Phi_{P}(\beta)\right)\right\}$ is the probability factor of not having any semihard activity in the underlying hadronic event. A more detailed analysis of the origin of the survival factor and of the suppression factor due to some elastic scattering is presented in the Appendix, in the context of a multiparton dynamics. In the same Appendix the influence of the possible deviation from the Poissonian form of the original parton distribution is also discussed. 


\section{Discussion and conclusions}

High energy hadronic interactions, with production of minijets and associated rapidity gap in the distribution of secondaries, is a process where one would expect to observe explicitly the unitarity relation at the level of partonic amplitudes. A closer inspection shows that the unitarity problem which one faces is twofold. On one hand, if one believes that the semihard interaction between partons can be described with the BFKL approach, one needs to solve the problem of the too rapid rise of the partonic cross section as a function of the rapidity interval between primary minijets. On the other hand, even if the size of the partonic cross section is not too large, namely if the hadronic c.m. energy is such that the typical rapidity interval between primary minijets is relatively small, the semihard hadronic cross section may still be too large. One can therefore distinguish three different regimes:

I- The cutoff is sizeable with respect to the typical energy available to the semihard partonic interaction. The corresponding 'elementary' parton interaction is small, no unitarization is needed and the semihard cross section is well described by a single partonic collision.

II- The cutoff is moved towards relatively smaller values, with respect to the available energy. A single partonic interaction is still well described by the BFKL dynamics. The semihard hadronic cross section is however too large with respect to the total inelastic cross section and unitarity corrections are to be taken into account. The unitarization of the hadronic semihard cross section is achieved by taking into account multiparton interactions, namely different pairs of partons interacting independently with BFKL Pomeron exchange. Typically the different partonic interactions are localized at different points in the transverse plane, in the region of overlap of the matter distribution of the two hadrons.

III- With even smaller values of the cutoff one may still be in the regime where perturbative QCD can be used, since the value of $\alpha_{S}\left(q_{t}^{\text {min }}\right)$ is small, but the typical rapidity interval between primary minijets is too large so that the 'elementary' parton process is not well described any more by the single BFKL Pomeron exchange and also the 'elementary' parton process has to be unitarized. One may obtain an indication on the limits between regions II and III by testing whether the bound in Eq.(15) is satisfied. 
To have a quantitative indication on the boundaries of the kinematical regions, we have worked out a numerical example. Since the present available information on multiple parton interactions is limited to the scale factor which gives the rate of double parton interactions[17], while no information is available on the multiparton correlations, we have considered the simplest possibility, namely the Poisson distribution for the multiparton distributions. On the grounds that the main contribution to the multiple parton interactions is represented by the disconnected partonic collisions, we have neglected parton rescatterings. The unitarized expression for the cross section is therefore given explicitly as a function of the input which is used to evaluate the single scattering term, namely the average number of partons $D(b, x)$ and the 'elementary' partonic cross section $\hat{\sigma}_{H}$. We have factorized $D(b, x)$ as $f_{\text {eff }}(x) \times F(b)$, where $f_{\text {eff }}(x)$ is the effective structure function as expressed in Eq.(6) and $F(b)$ is a Gaussian, normalized to one and such as to give for the double scattering term the scale factor $\sigma_{\text {eff }}$ (see Eq.(26) of Ref.[6]) consistent with the experimental indication[17]. In our numerical example we have chosen $\sigma_{e f f}=20 \mathrm{mb}$ and as a scale factor for the structure functions we have taken $q_{t}^{\min } / 2 . \alpha_{S}$ is a free parameter in the BFKL approach, one expects however that the value of $\alpha_{S}$ which one should use is not too different from the value of the running $\alpha_{S}$ at the scale of the typical momentum transferred in the process. We have chosen as a value of $\alpha_{S}$ the value of the running coupling computed with $q_{t}^{\min } / 2$ as a scale factor. The values of the semihard cross section $\sigma_{H}$, as expressed in Eq.(23), which we obtain with this input are consistent with the experimental values published by UA1[18]. At each value of the hadronic c.m. energy the boundary of the kinematical regions I, II and III are identified by the choice of the cutoff $q_{t}^{\min }$. The curve which corresponds to larger values of $q_{t}^{\min }$ in fig.1 has been drawn requiring that the unitarized hadronic semihard cross section $\sigma_{H}$, as expressed in Eq.(23), is $20 \%$ smaller with respect to the single scattering term. The lower curve corresponds to the value of $q_{t}^{\min }$ which, on the average, namely after integration with the structure functions, saturate the bound in Eq.(15). The two curves identify the three regions mentioned above.

Moving from large values of $q_{t}$ to the semihard region one faces therefore two different unitarity problems, which signal the appearance of different levels of structure in the hadronic interaction. The gap in the rapidity distribution of produced secondaries is an effect which derives from the unitarity relation applied to the 'elementary' parton amplitude. The regime where 'elementary' interactions 
with rapidity gap are a sizeable component of the 'elementary' parton process is however a regime where the overall hadronic process is already structured in a non-trivial way, because of the large amount of multiparton interactions. To have a quantitative feeling of the effect of multiparton interactions, we have performed a numerical calculation in the simplest example already considered to obtain the curves in fig.1. The expression of the cross section as a function of the rapidity interval $\Delta y$ is given in Eq.(29). We have considered $p \bar{p}$ interactions at Tevatron energy and as a lower threshold to observe minijets we have taken the value $q_{t}^{\text {min }}=$ $5 G e V$. In fig. 2 we plot the cross section, as expressed in Eq.(29), divided by the survival probability factor $\exp \left\{-\Phi_{S}(\beta)-\Phi_{P}(\beta)\right\}$. The continuous curve is obtained by using as a input the value $\sigma_{e f f}=20 \mathrm{mb}$ and the dashed curve is obtained by using $\sigma_{e f f}=12 \mathrm{mb}$. The dotted curve is the contribution of the single scattering term alone.

As it is shown in fig.2 the effect of unitarization on the behaviour of the cross section is large. In the actual case the main modification to the dependence on $\Delta y$ is due to the presence of multiple 'elastic' parton scatterings whose effect on the cross section is twofold. A different dependence on $\Delta y$, with respect to the single scattering term, is induced by the presence of two different sources. The first is the contribution of the process where the two observed minijets originate in different 'elastic' partonic interactions, the second term in Eq.(29), the second source for the different dependence on $\Delta y$ is the correction induced by multiple 'elastic' scatterings to the survival probability factor. In fact not all underlying hadron activity needs to be excluded. 'Elastic' parton scatterings which produce minijets outside the gap are allowed and the corresponding contribution to the cross section depends on $\Delta y$. The effect of the inelastic semihard partonic interactions is, on the contrary, factorized at fixed impact parameter $\beta$ and independent on $\Delta y$. The main effect of the inelastic partonic processes is to contribute to the survival probability $\left\langle S^{2}\right\rangle$ of ref.[4] rather than modifying the dependence on $\Delta y$.

The region where $q_{t}^{\text {min }}$ is relatively small is also the region which is closer to the BFKL kinematics and where, as a consequence, the BFKL approach to parton dynamics is better justified. In high energy hadronic interactions, at relatively small values of $q_{t}^{\text {min }}$, unitarity corrections are however large and have to be taken into account. The physical effect which underlies the need of unitarization is the increasing complexity of the semihard interaction which appears when $q_{t}^{\text {min }}$ is lowered, as it is shown in fig.1. In the kinematical region which has been discussed in 
the present paper, corresponding with region II in fig.1, the 'elementary' parton process is well described within the BFKL approach. The typical hadronic interaction is however characterized by several 'elementary' partonic collisions, localized at different points in the transverse plane. One of the reasons of interest in the actual kinematical regime is that, in region II, the non perturbative component of the process, which is factorized in the multiparton distributions, represents a new piece of information on the hadron structure with respect to the hadron structure functions of large $p_{t}$ physics.

\section{Appendix}

\section{A.1 General features and inelastic scattering}

In this Appendix the way in which an overall term independent of $\Delta y$ and further corrections explicitly dependent on $\Delta y$ may arise out of the many parton dynamics is discussed more in detail. The functional formulation, which has been already employed, is used again and more systematically. The longitudinal momenta are always given in terms of the fractional momentum $x$, the relation with the corresponding rapidity $y$ was mentioned in Sec.2.

If we look to configurations where there are two radiated partons (jets) with rapidity gap sitting in the central rapidity region, in the two-hadron c.m., then the two partons belong to different original hadrons. The parton population is divided into three segments $x_{a}, x_{b}, x_{c}$ : the $x_{a}$ interval correspond to the partons which would fill the gap, so to the parton that, in the configuration looked for, are not scattered; the $x_{b}$ correspond to the partons outside the gap, so to the partons which are simply not observed; the $x_{c}$ correspond to the parton observed at one end of the gap; the segment in which $x_{c}$ lies will be ideally shrunk to one point. The three regions of variations of $x$ reflect into three regions of variations of $u$, but clearly the impact parameter $\mathbf{b}$ is not affected by this operation.

This decomposition suggests a way of rewriting the generating functional 
which is sometimes more convenient :

$$
\begin{aligned}
\mathcal{Z}[J] & =\sum_{n} \frac{1}{n !} \int J\left(u_{1}\right) \cdots J\left(u_{n}\right) W_{n}\left(u_{1}, \ldots u_{n}\right) d u_{1} \ldots d u_{n} \\
& =\sum_{p, q, k} \frac{1}{p !} \frac{1}{q !} \frac{1}{k !} \int J\left(u_{a}\right) \ldots J\left(u_{b}\right) \ldots J\left(u_{c}\right) W_{p, q, k}\left(u_{a}, \ldots u_{b}, \ldots u_{c}\right) d u_{a} \ldots d u_{b} \ldots d u_{c},
\end{aligned}
$$

$p$ is the number of partons of kind $a, q$ is the number of partons of kind $b, k$ is the number of partons of kind $c$, and an analogous expression is written for $\mathcal{Z}\left[I\left(u^{\prime}\right)\right]$.

We start considering the effect of inelastic processes and we express the probability that:

I- A parton $u_{c}$ scatters elastically against a parton $u_{c}^{\prime}$ or a parton $u_{c}$ scatters elastically against a parton $u_{b}^{\prime}$ and parton $u_{c}^{\prime}$ scatters elastically against a parton $u_{b}$ thus giving rise to the two partons at the end of the gap and nothing is produced in the middle.

II- No inelastic scattering takes place, since such a process would give rise also to partons which could fill the gap.

In formulae the requirement $\mathrm{I}$ is expressed by an operator $\mathcal{F}$ while the requirement II is expressed by an operator $\mathcal{G}$, both applied to the product $\mathcal{Z}[J] \mathcal{Z}[I]$.

The actual form of the two operators is:

$$
\begin{aligned}
& \mathcal{F}= \int d u_{c} d u_{c}^{\prime}\left[\delta_{c}(u) \hat{\sigma}_{E}\left(u_{c}, u_{c}^{\prime}\right) \delta_{c}^{\prime}\left(u^{\prime}\right)+\right. \\
&\left.\left(\int \delta_{c}(u) \hat{\sigma}_{E}\left(u_{c}, u_{b}^{\prime}\right) \delta_{b}^{\prime}\left(u^{\prime}\right) \delta_{b}(u) \hat{\sigma}_{E}\left(u_{b}, u_{c}^{\prime}\right) \delta_{c}^{\prime}\left(u^{\prime}\right) d u_{b} d u_{b}^{\prime}\right)\right] \\
& \mathcal{G}=\sum_{n, n^{\prime}} \iint \frac{1}{n !} \frac{1}{n^{\prime} !} \delta_{1}(u) \cdots \delta_{n}(u) \cdots \delta_{1}^{\prime}\left(u^{\prime}\right) \cdots \delta_{n^{\prime}}^{\prime}\left(u^{\prime}\right) \times \\
& \prod\left[1-\hat{\sigma}_{I n}\left(u, u^{\prime}\right)\right] d u d u^{\prime}
\end{aligned}
$$

In the definition of $\mathcal{G}$ there is no point in distinguishing the intervals of rapidity. Some notational simplification is obtained by setting:

$$
\frac{\delta}{\delta J\left(u_{m}\right)}=\delta_{m}(u) \quad \frac{\delta}{\delta I\left(u_{m}^{\prime}-\beta\right)}=\delta_{m}^{\prime}\left(u^{\prime}\right) \quad m=a, b .
$$


A first step in the choice of some specific distribution could be to neglect the genuine many-body correlations, in this case the generating functional is in fact reduced to an ordinary function of a linear functional of the sources

$$
\mathcal{Z}[J]=\Phi(\mathcal{Y}) \quad \mathcal{Y}=\int D(u) J(u) d u
$$

The division of the field of variation of $x$ into three parts induces a corresponding decomposition $\mathcal{Y}=\mathcal{Y}_{a}+\mathcal{Y}_{b}+\mathcal{Y}_{c}$.

Even with the restriction to a pure one-body density the general expression is complicated. A very relevant and effective simplification is produced if we assume a Poissonian distribution for the initial partonic distributions

$$
\mathcal{Z}[J]=\exp \left[\mathcal{Y}-\mathcal{Y}_{o}\right]
$$

The normalization term $\mathcal{Y}_{o}=\int D(u) d u$ ensures that $\mathcal{Z}[1]=1$.

In looking for some general features of the inelastic rescattering it is however possible to use a general partonic distribution without many-body correlations, as it is described by the generating functional $\Phi(\mathcal{Y})$; so we let the functional differential operators $\mathcal{G}$ and $\mathcal{F}$ act on the product $\Phi(\mathcal{Y}[J]) \Phi(\mathcal{Y}[I])$ at the end the auxiliary sources are put to zero: $J=I=0$. From the action of the operator $\mathcal{G}$ one obtains the intermediate result:

$$
\begin{gathered}
\mathcal{K}[J, I]=\sum_{n, n^{\prime}} \iint \frac{1}{n !} \frac{1}{n^{\prime} !} D\left(u_{1}\right) \cdots D\left(u_{n}\right) \cdots D\left(u_{1}^{\prime}-\beta\right) \cdots D\left(u_{n^{\prime}}^{\prime}-\beta\right) \times \\
\prod\left[1-\hat{\sigma}_{I n}\left(u, u^{\prime}\right)\right] d u d u^{\prime} \Phi^{(n)}(\mathcal{Y}[J]) \Phi^{\left(n^{\prime}\right)}(\mathcal{Y}[I])
\end{gathered}
$$

From the further action of the operator $\mathcal{F}$ and the condition $J=I=0$ one obtains the final result:

$$
\begin{aligned}
K(\beta)= & \sum_{n, n^{\prime}} \iint \frac{1}{n !} \frac{1}{n^{\prime} !} D\left(u_{1}\right) \cdots D\left(u_{n}\right) \cdots D\left(u_{1}^{\prime}-\beta\right) \cdots D\left(u_{n^{\prime}}^{\prime}-\beta\right) \\
& \prod\left[1-\hat{\sigma}_{I n}\left(u, u^{\prime}\right)\right] d u d u^{\prime} \times \\
& \int d u_{c} d u_{c}^{\prime}\left[D\left(u_{c}\right) \hat{\sigma}_{E}\left(u_{c}, u_{c}^{\prime}\right) D\left(u_{c}^{\prime}-\beta\right) \Phi^{(n+1)}(0) \Phi^{\left(n^{\prime}+1\right)}(0)+\right. \\
& \int D\left(u_{c}\right) \hat{\sigma}_{E}\left(u_{c}, u_{b}^{\prime}\right) D\left(u_{b}^{\prime}-\beta\right) D\left(u_{b}\right) \hat{\sigma}_{E}\left(u_{b}, u_{c}^{\prime}\right) D\left(u_{c}^{\prime}-\beta\right) d u_{b} d u_{b}^{\prime} \\
& \left.\Phi^{(n+2)}(0) \Phi^{\left(n^{\prime}+2\right)}(0)\right]
\end{aligned}
$$


So in general the expression has the form

$$
K(\beta)=\sum_{n, n^{\prime}}\left[G_{n, n^{\prime}}^{I}(\beta) \cdot F^{I}(\beta)+G_{n, n^{\prime}}^{I I}(\beta) \cdot F^{I I}(\beta)\right]
$$

For a Poissonian partonic distribution it results for every derivative $\Phi^{(n)}(0)=$ $\exp \left[-\mathcal{Y}_{o}\right]$, so the double sum over $n, n^{\prime}$ can in principle be carried out yielding an overall factor $G(\beta)=\sum_{n, n^{\prime}}\left[G_{n, n^{\prime}}^{I}(\beta)+G_{n, n^{\prime}}^{I I}(\beta)\right]$ which multiplies the remaining term

$$
\begin{aligned}
F(\beta)= & \int d u_{c} d u_{c}^{\prime}\left[D\left(u_{c}\right) \hat{\sigma}_{E}\left(u_{c}, u_{c}^{\prime}\right) D\left(u_{c}^{\prime}-\beta\right)+\right. \\
& \left.\int D\left(u_{c}\right) \hat{\sigma}_{E}\left(u_{c}, u_{b}^{\prime}\right) D\left(u_{b}^{\prime}-\beta\right) D\left(u_{b}\right) \hat{\sigma}_{E}\left(u_{b}, u_{c}^{\prime}\right) D\left(u_{c}^{\prime}-\beta\right) d u_{b} d u_{b}^{\prime}\right] .
\end{aligned}
$$

In other words it results that, at fixed hadronic impact parameter $\beta$ the inelastic processes give simply rise to a multiplicative factor to the fundamental amplitude.

In presence of another kind of distribution the treatment is less straightforward because the sum over $n, n^{\prime}$ does not allows the extraction of a common term $F(\beta)$. It seems however likely that in the expression of $K$ the second addendum, containing $F^{I I}$ is more important than the first one, because it involves a less exclusive condition, if this is true then the sum

$$
\bar{G}(\beta)=\sum_{n, n^{\prime}} G_{n, n^{\prime}}^{I I}(\beta)
$$

can be still be carried out yielding again a multiplicative factor in front of the fundamental amplitude at fixed hadronic impact parameter.

This essential simplicity is destroyed if we are in presence of sizeable twobody correlations. To be definite we may consider an example where we have a Poissonian distribution corrected by a two-body correlation

$$
\begin{gathered}
\mathcal{Z}[J]=\exp \left[\mathcal{Y}-\mathcal{Y}_{o}\right] \\
\mathcal{Y}=\int D(u) J(u) d u+\frac{1}{2} \int C(u, \bar{u}) J(u) J(\bar{u}) d u d \bar{u} .
\end{gathered}
$$

Then in performing the derivatives in order to calculate $K(\beta)$ we end unavoidably with expressions where the correlation term $C(u, \bar{u})$ links the inelastic component 
with the elastic fundamental one and so the decomposition given in Eq.(A.10) is no longer possible, in this case we expect that the inelastic processes modify strongly not only the size but also the shape of the fundamental elastic process.

\section{A.2 Effect of the multiple elastic scattering}

It is clear that in presence of a large flux of partons the inelastic processes that have been considered till now are not the only disturbing effects i.e. there are other dynamical processes which also act in the sense of filling the rapidity gap that the "fundamental" process described in the present formalism by $\mathcal{F}$ produces. With reference to the notation introduced in eq (A.1), one must take into account also the following elastic processes:

A parton $u_{a}$ which scatters against a parton $u_{a}^{\prime}$, this event will fill the gap. A parton $u_{a}$ which scatters against a parton $u_{b}^{\prime}$ or a parton $u_{b}$ which scatters against a parton $u_{a}^{\prime}$, this event will also fill the gap. The statement that these kind of collisions must not happen is expressed by a veto operator which, in absence of inelastic interactions would have the following form:

$$
\begin{aligned}
\mathcal{E}=\sum_{p, p^{\prime}} \sum_{q, q^{\prime}} \iint \frac{1}{p !} \frac{1}{q !} \frac{1}{p^{\prime} !} \frac{1}{q^{\prime} !} \delta_{a}(u) \cdots \delta_{b}(u) \cdots \delta_{a}^{\prime}\left(u^{\prime}\right) \cdots \delta_{b}^{\prime}\left(u^{\prime}\right) \times \\
\prod\left[1-\hat{\sigma}_{E}\left(u_{a}, u_{a}^{\prime}\right)\right] \cdot\left[1-\hat{\sigma}_{E}\left(u_{a}, u_{b}^{\prime}\right)\right] \cdot\left[1-\hat{\sigma}_{E}\left(u_{b}, u_{a}^{\prime}\right)\right] d u_{a} d u_{b} d u_{a}^{\prime} d u_{b}^{\prime}
\end{aligned}
$$

The fact that the parton of kind $b$ are unobserved suggests that the sum over $q, q^{\prime}$ is the first operation to be performed.

For a fixed $p$ the term containing the sum over $q^{\prime}$ is

$$
\begin{aligned}
& \Delta\left(u_{a, 1}, \cdots, u_{a, p}\right)=\delta_{a}\left(u_{1}\right) \cdots \delta_{a}\left(u_{p}\right) \times \\
& \sum_{q^{\prime}} \frac{1}{q^{\prime} !} \prod_{k}\left[\int\left[1-\hat{\sigma}_{E}\left(u_{a, 1}, u_{k}^{\prime}\right)\right] \cdots\left[1-\hat{\sigma}_{E}\left(u_{a, p}, u_{k}^{\prime}\right)\right] \delta_{b}^{\prime}\left(u_{k}^{\prime}\right) d u_{k}^{\prime}\right] .
\end{aligned}
$$

Since all the $u_{k}^{\prime}$ are dummy variables the sum over $q^{\prime}$ can be carried out and gives an exponential; then the product of the $[1-\hat{\sigma}]$ factors in the exponent may be expanded in terms containing no $\hat{\sigma}$, one $\hat{\sigma}$, two $\hat{\sigma}$, and so on.

$$
\begin{aligned}
& \Delta\left(u_{a, 1}, \cdots, u_{a, p}\right)=\delta_{a}\left(u_{1}\right) \cdots \delta_{a}\left(u_{p}\right) \times \\
& \quad \exp \left[\int\left(1-\sum_{j} \hat{\sigma}_{E}\left(u_{a, j}, u_{k}^{\prime}\right)+\sum_{i \neq j} \frac{1}{2} \hat{\sigma}_{E}\left(u_{a, i}, u_{k}^{\prime}\right) \hat{\sigma}_{E}\left(u_{a, j}, u_{k}^{\prime}\right)+\cdots\right) \delta_{b}^{\prime}\left(u_{k}^{\prime}\right) d u_{k}^{\prime}\right],
\end{aligned}
$$


The sum over $q$ is obviously treated in the same way and we get for $\mathcal{E}$ the expression:

$$
\mathcal{E}=\sum_{p, p^{\prime}} \int \frac{1}{p !} \frac{1}{p^{\prime} !} \prod\left[1-\hat{\sigma}_{E}\left(u_{a}, u_{a}^{\prime}\right)\right] \Delta\left(u_{a, 1}, \cdots, u_{a, p}\right) \Delta\left(u_{a, 1}^{\prime}, \cdots, u_{a, p^{\prime}}^{\prime}\right) \prod d u_{a} d u_{a}^{\prime}
$$

In order to obtain more explicit expressions it is necessary, now, to expand both the operators $\Delta$ and the term $\prod[1-\hat{\sigma}]$ in multiple interactions, but at this step it seems that the treatment becomes clearer if one chooses some definite form of the parton distributions.

We choose now a Poissonian distribution of partons * and let the functional differential operator $\mathcal{E}$ act on the product $\mathcal{Z}[J] \mathcal{Z}[I]$, after the action of $\mathcal{F}$. Only at the end the auxiliary sources are put to zero: $J=I=0$. As it was already said, for the operator $\mathcal{E}$ one is forced to proceed in steps corresponding to successive reinteractions of the same partons: from the operator $\Delta$ one gets:

$$
\begin{aligned}
& A\left(u_{a, 1}, \cdots, u_{a, p}\right)=D\left(u_{a, 1}\right) \cdots D\left(u_{a, p}\right) \times \\
& \quad \exp \left[\int\left[1-\sum_{i} \hat{\sigma}_{E}\left(u_{a, i}, u_{b}^{\prime}\right)+\sum_{i \neq j} \frac{1}{2} \hat{\sigma}_{E}\left(u_{a, i}, u_{b}^{\prime}\right) \hat{\sigma}_{E}\left(u_{a, j}, u_{b}^{\prime}\right)+\cdots\right] D\left(u_{b}^{\prime}-\beta\right) d u_{b}^{\prime}\right] .
\end{aligned}
$$

Also the term $P=\prod\left[1-\hat{\sigma}_{E}\left(u_{a, i}, u_{a, r}^{\prime}\right)\right] \equiv \prod\left[1-\hat{\sigma}_{i, r}\right]$ is expanded with the result:

$$
P=1-\sum \hat{\sigma}_{i, r}+\sum \sum \frac{1}{2} \hat{\sigma}_{i, r} \hat{\sigma}_{j, s}-\sum \sum \sum(1 / 3 !) \hat{\sigma}_{i, r} \hat{\sigma}_{j, s} \hat{\sigma}_{k, t}+\cdots
$$

In the repeated sums the pairs of indices cannot be equal e.g. $(i, r) \neq(j, s)$; it is however possible to have either $i=j$ or $r=s$, what correspond to a rescattering of a particular parton. If we took only the term 1 and the simple sum in the exponent of $\Delta$, out of the operator $\mathcal{E}$ we would get this result:

$$
\begin{aligned}
E_{o}= & \exp \left[\int D\left(u_{a}\right) \Gamma\left(u_{a}-\beta\right) d u_{a}\right] \exp \left[\int D\left(u_{a}^{\prime}-\beta\right) \Gamma\left(u_{a}^{\prime}\right) d u_{a}^{\prime}\right] \times \\
& \exp \left[\int D\left(u_{b}\right) d u_{b}+\int D\left(u_{b}^{\prime}\right) d u_{b}^{\prime}\right] .
\end{aligned}
$$

* In order to perform the actual calculation it may be useful to remember the identity $\left.f(d / d x) g(x)\right|_{x=0}=\left.g(d / d x) f(x)\right|_{x=0}$, which is evident whenever the two functions admit a power expansion around 0 ; in particular it yields $\left.f(d / d x) \mathrm{e}^{h x}\right|_{x=0}=$ $f(h)$ 
In this formula the absorption factors $\Gamma$ have been introduced, in their definition the property that $\hat{\sigma}$ depends only on the difference of the impact parameters has been used.

$$
\begin{aligned}
\Gamma\left(u_{a}-\beta\right) & =\exp \left[-\int \hat{\sigma}_{E}\left(u_{a}, u_{b}^{\prime}\right) D\left(u_{b}^{\prime}-\beta\right) d u_{b}^{\prime}\right] \\
\Gamma\left(u_{a}^{\prime}\right) & =\exp \left[-\int \hat{\sigma}_{E}\left(u_{a}^{\prime}, u_{b}\right) D\left(u_{b}\right) d u_{b}^{\prime}\right] .
\end{aligned}
$$

The factor $E_{o}$ takes into account the interaction of the partons of kind $a$ with those of kind $b$, but not the interaction of the $a$-partons among themselves. It could be put in a form that could be of easier interpretation. i.e.

$$
\begin{aligned}
E_{o}= & \exp \left[\int D(u) d u+\int D\left(u^{\prime}\right) d u^{\prime}\right] \times \\
& \exp \left[-\int D\left(u_{a}\right)\left[1-\Gamma\left(u_{a}-\beta\right)\right] d u_{a}\right] \exp \left[-\int D\left(u_{a}^{\prime}-\beta\right)\left[1-\Gamma\left(u_{a}^{\prime}\right)\right] d u_{a}^{\prime}\right] .
\end{aligned}
$$

In this expression the first exponential, where also the small contribution from $u_{c}$ has been included, takes simply away the normalization term $\exp \left[-\mathcal{Y}_{o}-\mathcal{Y}_{o}^{\prime}\right]$, it does not belong to the multiple scattering, while the second term, $E_{o}^{\prime}$ is an "absorption" term due to some of the disconnected collision which fill the gap. We can do better and consider the other terms in the expansion of $P=\prod\left[1-\hat{\sigma}_{i, j}\right]$, with the restriction of excluding the repeated interaction of the same $a$-parton, in formulae $i \neq j$ and $r \neq s$ in this way a further factor is produced which multiply the former expression, i.e.

$$
E_{1}=\exp \left[-\int D\left(u_{a}\right) \Gamma\left(u_{a}-\beta\right) \hat{\sigma}_{E}\left(u_{a}, u_{a}^{\prime}\right) D\left(u_{a}^{\prime}-\beta\right) \Gamma\left(u_{a}^{\prime}\right) d u_{a} d u_{a}^{\prime}\right]
$$

Going on means to produce expressions quite elaborated, one could, as an example, calculate the factors which describe a double interaction of partons either of kind $a$ or of kind $b$. When the partons $a$ are allowed to collide twice, but not three times, the following factor is produced:

$$
\begin{gathered}
E_{2}=\exp \frac{1}{2}\left[\int D\left(u_{a}\right) \Gamma\left(u_{a}-\beta\right) \hat{\sigma}_{E}\left(u_{a}, u_{a}^{\prime}\right) D\left(u_{a}^{\prime}-\beta\right) \times\right. \\
\Gamma\left(u_{a}^{\prime}\right) \hat{\sigma}_{E}\left(u_{a}^{\prime}, u_{\bar{a}}\right) D\left(u_{\bar{a}}\right) \Gamma\left(u_{\bar{a}}-\beta\right) d u_{a} d u_{\bar{a}} d u_{a}^{\prime}+ \\
\int D\left(u_{a}^{\prime}-\beta\right) \Gamma\left(u_{a}^{\prime}\right) \hat{\sigma}_{E}\left(u_{a}^{\prime}, u_{a}\right) D\left(u_{a}\right) \times \\
\left.\Gamma\left(u_{a}-\beta\right) \hat{\sigma}_{E}\left(u_{a}, u_{\bar{a}}^{\prime}\right) D\left(u_{\bar{a}}^{\prime}-\beta\right) \Gamma\left(u_{\bar{a}}^{\prime}\right) d u_{a} d u_{a}^{\prime} d u_{\bar{a}}^{\prime}\right] .
\end{gathered}
$$


The expansion can be further continued, e.g. by considering also the collision of two partons of kind $b$ and then the various results can be collected. From the term explicitly calculated one gets: $E \approx E_{o}^{\prime} E_{1} E_{2} \cdots$. This factor modifies qualitatively the result expressed in Eq.(A.9): because in that expression the terms $G_{n, n^{\prime}}$ were independent of the rapidities defining the gap which appear in $F_{n, n^{\prime}}$ through $u_{c}=\left(x_{c}, \mathbf{b}\right)$; now, on the contrary these variables appear explicitly in the terms $E$.

Other form of partonic distributions give rise to more complicated final expressions, but some of them allow anyhow some elaboration, in particular as already remarked in [14], the generating functional of a negative binomial distribution allow a representation which is an integral transform of a Poisson generating functional:

$$
\Phi(\mathcal{Y})=\frac{\left(1-\mathcal{Y}_{o}\right)^{\alpha}}{(1-\mathcal{Y})^{\alpha}}=\frac{1}{\Gamma(\alpha)}\left(1-\mathcal{Y}_{o}\right)^{\alpha} \int \mathrm{e}^{t \mathcal{Y}} \mathrm{e}^{-t} t^{\alpha-1} d t
$$

So, in principle, from the previous expressions holding for a Poisson distribution of partons it would be possible to get the analogous one for a negative binomial distribution by applying the following prescriptions:

I-Take away the normalization term, $\mathcal{N}_{P}=\exp \left[-\mathcal{Y}_{o}-\mathcal{Y}_{o}^{\prime}\right]$ and substitute it by

$$
\mathcal{N}_{B}=[\Gamma(\alpha)]^{-2}\left(1-\mathcal{Y}_{o}\right)^{\alpha}\left(1-\mathcal{Y}_{o}^{\prime}\right)^{\alpha}
$$

II-Multiply by a factor $t$ every $D(u)$ explicit or implicit, i.e. inside $\Gamma$ and $B$;

and multiply by a factor $s$ every $D\left(u^{\prime}\right)$ explicit or implicit, i.e. inside $\Gamma$ and $B$.

III-Multiply the overall resulting expression by $\mathrm{e}^{-t-s}(t s)^{\alpha-1}$.

IV-Integrate the result in $d t d s$ from 0 to $+\infty$.

It is evident that the Poissonian and the negative binomial distribution for the original partons are only the simplest ones which can be chosen; in term of integral transforms of the generating functions other distributions could be built up.

\section{A.3 Elastic and inelastic scattering}

The real case implies evidently both elastic and inelastic scattering. The requirement that there be no production process filling the gap is expressed by an operator of the form 


$$
\begin{gathered}
\mathcal{C}=\sum_{p, p^{\prime}} \sum_{q, q^{\prime}} \iint \frac{1}{p !} \frac{1}{q !} \frac{1}{p^{\prime} !} \frac{1}{q^{\prime} !} \delta_{a}(u) \cdots \delta_{b}(u) \cdots \delta_{a}^{\prime}\left(u^{\prime}\right) \cdots \delta_{b}^{\prime}\left(u^{\prime}\right) \times \\
\prod\left[1-\hat{\sigma}_{T}\left(u_{a}, u_{a}^{\prime}\right)\right] \cdot\left[1-\hat{\sigma}_{T}\left(u_{a}, u_{b}^{\prime}\right)\right] \cdot\left[1-\hat{\sigma}_{T}\left(u_{b}, u_{a}^{\prime}\right)\right] \\
\prod\left[1-\hat{\sigma}_{I n}\left(u_{b}, u_{b}^{\prime}\right)\right] d u_{a} d u_{b} d u_{a}^{\prime} d u_{b}^{\prime} \\
\hat{\sigma}_{T}=\hat{\sigma}_{E}+\hat{\sigma}_{I n}
\end{gathered}
$$

The statement expressed by this operator is that while the parton of kind $a$ must not suffer interactions at all, the partons of kind $b$ may interact provided their interaction is elastic. In its full form this expression appears quite intractable, however one can follow the idea that the most relevant dynamical feature is the inelastic scattering and the elastic is a perturbation. It does not seems convenient, however, to start from the inelastic operator $\mathcal{G}$, it is more efficient to start with an "exaggerated" form of the operator $\mathcal{C}$ such that also the elastic interactions of the partons $b$ are excluded, by defining

$$
\mathcal{C}_{o}=\sum_{n, n^{\prime}} \iint \frac{1}{n !} \frac{1}{n^{\prime} !} \delta_{1}(u) \cdots \delta_{n}(u) \cdots \delta_{1}^{\prime}\left(u^{\prime}\right) \cdots \delta_{n^{\prime}}^{\prime}\left(u^{\prime}\right) \prod\left[1-\hat{\sigma}_{T}\left(u, u^{\prime}\right)\right] d u d u^{\prime}
$$

which looks very similar to $\mathcal{G}$, eq(A.4) and so yields, by itself, a factor independent of $y$. Then one has to correct the result by an expansion in $\hat{\sigma}_{E}$ that uses eq (A.23). Since the coordinates $u$ are all equivalent the expansion has the same combinatorial structure as:

$$
[A+B]^{q q^{\prime}}=A^{q q^{\prime}}+q q^{\prime} B A^{(q-1)\left(q^{\prime}-1\right)} \cdot A^{q-1} \cdot A^{q^{\prime}-1}+B^{2} \ldots
$$

In this way, through a shift in $q$ and $q^{\prime}$, the integrand of the operator $\mathcal{C}_{o}$ is reproduced with additional factors. The actual form of the first order correction in $\hat{\sigma}_{E}$ is:

$$
\begin{aligned}
\mathcal{C}_{1}= & \sum_{p, p^{\prime}} \sum_{q, q^{\prime}} \iint \frac{1}{p !} \frac{1}{q !} \frac{1}{p^{\prime} !} \frac{1}{q^{\prime} !} \delta_{a}(u) \cdots \delta_{b}(u) \cdots \delta_{a}^{\prime}\left(u^{\prime}\right) \cdots \delta_{b}^{\prime}\left(u^{\prime}\right) \delta_{b}(v) \delta_{b}^{\prime}\left(v^{\prime}\right) \times \\
& \prod\left[1-\hat{\sigma}_{T}\left(u_{a}, u_{a}^{\prime}\right)\right] \cdot\left[1-\hat{\sigma}_{T}\left(u_{a}, u_{b}^{\prime}\right)\right] \cdot\left[1-\hat{\sigma}_{T}\left(u_{b}, u_{a}^{\prime}\right)\right] \cdot\left[1-\hat{\sigma}_{T}\left(u_{b}, u_{b}^{\prime}\right)\right] \times \\
& d u_{a} d u_{b} d u_{a}^{\prime} d u_{b}^{\prime} \\
& \int \hat{\sigma}_{E}\left(v_{b}, v_{b}^{\prime}\right) \prod\left[1-\hat{\sigma}_{T}\left(u_{b}, v_{b}^{\prime}\right)\right] \prod\left[1-\hat{\sigma}_{T}\left(v_{b}, u_{b}^{\prime}\right)\right] \delta_{b}(v) \delta_{b}^{\prime}\left(v^{\prime}\right) d v_{b} d v_{b}^{\prime} .
\end{aligned}
$$


In a limit in which terms like $\hat{\sigma}_{E} \cdot \hat{\sigma}_{T}$ are neglected, while keeping the powers of $\hat{\sigma}_{T}$ the correction may be factorized in the form

$$
\mathcal{C}_{1} \approx \mathcal{C}_{o} \cdot \int \hat{\sigma}_{E}\left(v_{b}, v_{b}^{\prime}\right) \delta_{b}(v) \delta_{b}^{\prime}\left(v^{\prime}\right) d v_{b} d v_{b}^{\prime}
$$

This approximation for the correction $\mathcal{C}_{1}$ suggests also a generalization. The expression itself originates from the division of the whole flux of parton in a pair suffering only elastic scattering and in a rest for which all interaction are considered. This attitude can be extended to more general situation, on the ground that, as already noticed, in presence of large parton fluxes the multiple disconnected interactions may become more relevant than the reinteractions. So, with reference to Eq.(A.22) we can divide the $q$ partons of kind $b$ into two subsets of $r$ and $s$ partons, obviously with $r+s=q$, and the same for $q^{\prime}$. The coordinates of the $r$ partons will be still denoted by $u$, the coordinates of the $s$ partons will be called $v$. In this way Eq.(A.22) takes the following form:

$\mathcal{C}=\sum_{p, p^{\prime}} \sum_{r, r^{\prime}} \sum_{s, s^{\prime}} \int \frac{1}{p !} \frac{1}{r !} \frac{1}{s !} \frac{1}{p^{\prime} !} \frac{1}{r^{\prime} !} \frac{1}{s^{\prime} !} \delta_{a}(u) \cdots \delta_{b}^{\prime}\left(v^{\prime}\right) \cdot \prod[1-\hat{\sigma}] d u_{a} d u_{b} d v_{b} d u_{a}^{\prime} d u_{b}^{\prime} d v_{b}^{\prime}$

the product $\Pi[1-\hat{\sigma}]$ contains nine kinds of factors. Now we decide, on the basis of the physical consideration outlined before that the $v$ parton suffer only elastic scattering, without any other interaction, then necessarily $s=s^{\prime}$, and there are $s$ ! ways of pairing the $v$ with the $v^{\prime}$. So it is not difficult to see that the rest of $\mathcal{C}$ reconstruct the a factor $\mathcal{C}_{o}$ of Eq.(15), whereas the sum over $s$ yields an exponential. In this way the approximate expression for $\mathcal{C}$ is given by:

$$
\mathcal{C} \approx \mathcal{C}_{o} \cdot \exp \left[\int \delta_{b}\left(v^{\prime}\right) \hat{\sigma}_{E}\left(v_{b}, v_{b}^{\prime}\right) \delta_{b}^{\prime}\left(v^{\prime}\right) d v_{b} d v_{b}^{\prime}\right]
$$

When the parton distribution is Poissonian out of Eq.(A.27') one gets the expression of the first correction due to multiple scattering depending on $\Delta y$ which corresponds to what was previously stated in section 3, Eq.(29). Anyhow, in a way wholly independent of the validity of the approximation we see that the correction is always positive because it represents a compensation for the previous exclusion of configurations that should be allowed, the amount of the correction diminishes by enlarging the rapidity gap since the integration runs over the complementary 
domain. For our purposes the most relevant feature of the elastic interactions is that they produce effects depending from the very beginning on the $y$ of the gap because the integrations over $v, v^{\prime}$ depend explicitly on $x_{c}, x_{c}^{\prime}$ which are precisely the variables that set the difference between partons of kind $a$ and partons of kind $b$. 


\section{References}

1. B.M. McCoy and T.T. Wu, Phys. Rev. Lett. 35, 604 (1975); Phys. Rev. D13, 1076 (1976); V.S. Fadin, E.A. Kuraev and L.N. Lipatov, Phys. Lett. B60, 50 (1975); L.Łukaszuk and X.Y. Pham, Phys. Lett. B53, 287 (1974); H.T. Nieh and Y.P. Yao, Phys. Rev. D13, 1082 (1976); L. Tyburski, Phys. Rev. D13, 1107 (1976); L.L. Frankfurt and V.E. Sherman, Sov. J. Nucl. Phys. 23, 581 (1976); C.Y. Lo and H. Cheng, Phys. Rev. D13, 1131 (1976); P.S. Yeung, Phys. Rev. D13, 2306 (1976); H. Cheng and C.Y. Lo, Phys. Rev. D15, 2959 (1977); H. Cheng and T.T. Wu, Expanding Protons: Scattering at High Energies, Cambridge, Mass., The MIT Press, 1987; H. Cheng, J.A. Dickinson and K. Olaussen, Phys. Rev. D23, 534 (1981).

2. M.T. Grisaru, H.J. Schnitzer and H.S. Tsao Phys. Rev. Lett 30, 811 (1973); L.N. Lipatov, Yad. Fiz. 23, 642 (1976); E.A. Kuraev, L.N. Lipatov and V.S. Fadin, Zh. Eksp. Teor. Fiz. 71, 840 (1976) [Sov. Phys. JEPT 44, 443 (1976)]; 72, 377 (1977) [Sov. Phys. JEPT 45, 199 (1977)]; Ya.Ya. Balitskii

and L.N. Lipatov, Yad. Fiz. 28, 1597 (1978) [Sov. J. Nucl. Phys. 28, 822 (1978)]; Pis'ma Zh. Eksp. Teor. Fiz. 30, 383 (1979) [JETP Lett. 30, 355 (1979)]; V. Del Duca, preprint DESY 95-023, DFTT 13/95 (unpublished); V.T. Kim and G.B. Pivovarov, Phys. Rev. D53, 6 (1996); Phys. Rev. D54, 725 (1996); M.A. Braun, Phys. Lett. B345, 155 (1995); Z. Phys. C70, 103 (1996); N.N. Nikolaev, G.B. Zakharov and V.R. Zoller, Phys. Lett. B366, 337 (1966).

3. V. Del Duca and W.-K. Tang, Phys. Lett. B312, 225 (1993).

4. J.D. Bjorken, Phys. Rev. D47, 101 (1992).

5. E. Gotsman, E.M. Levin and U. Maor, Phys. Lett. B353, 526 (1995).

6. R. Ragazzon and D. Treleani, Phys. Rev. D53, 55 (1996).

7. A.H. Mueller and W.-K. Tang, Phys. Lett. B284, 123 (1992).

8. L.V. Gribov, E.M. Levin and M.G. Ryskin, Phys. Rep. 100, 1 (1983).

9. V. Abramovskii, V.N. Gribov and O.V. Kancheli, Yad. Fiz. 18, 595 (1973) [Sov. J. Nucl. Phys. 18, 308 (1974) ].

10. L. Frankfurt, W. Koepf and M. Strikman Phys. Rev. D54, 3194 (1996).

11. G. Pancheri and Y. Srivastava Phys. Lett. B182, 199 (1986); S. Lomatch, F.I. Olness and J.C. Collins Nucl. Phys. B317, 617 (1989). 
12. M. Jacob and P.V. Landshoff, Mod. Phys. Lett. A1, 657 (1986); Ll. Ametller and D. Treleani, Int. J. Mod. Phys. A3, 521 (1988).

13. L.S. Brown Phys. Rev. D5, 748 (1972); Y. Akiyama, S. Hori Progr. Theor. Phys. 48, 276 (1973)

14. G. Calucci and D. Treleani, Nucl. Phys. B (Proc. Suppl.) 18C, 187 (1990); Int. J. Mod. Phys. A6, 4375 (1991).

15. G. Calucci and D. Treleani Phys. Rev. D49, 138 (1994); D50, 4703 (1994); J. Bartels and M. Wüsthoff, Z. Phys. C66, 157 (1995).

16. H.D. Politzer, Nucl. Phys. B172, 349 (1980); R.K. Ellis, R. Petronzio and W. Furmanski, ibid. B207, 1 (1981); N. Paver and D. Treleani, Nuovo Cimento A70, 215 (1982); Zeit. Phys.C28, 187 (1985); B. Humpert, Phys. Lett. 131B, 461 (1983); B. Humpert and R. Odorico, ibid 154B, 211 (1985); T. Sjostrand and M. Van Zijl, Phys. Rev. D36, 2019 (1987).

17. F. Abe et al., Phys. Rev. D47, 4857 (1993).

18. C. Albajar et al. Nucl. Phys. B309, 405 (1988). 


\section{Figure captions}

Fig.1- The three different kinematical regions which characterize semihard hadronic interactions. I: only the single partonic collision, described by a single BFKL Pomeron exchange, is relevant; II: multiparton collisions are to be taken into account, each partonic interaction is however well described by a single BFKL Pomeron exchange; III: the single BFKL Pomeron exchange is not any more an adequate description of the single parton interaction.

Fig.2- Cross section for production of minijets with rapidity gap as a function of the gap $\Delta y$. The process is $p \bar{p}$ at $\sqrt{s}=1.8 T e V$ and $q_{t}^{\text {min }}=5 G e V$. The dotted curve is the single scattering term. The continuous and the dashed curves include all disconnected multiple parton collisions. Input to the continuous curve is $\sigma_{e f f}=20 \mathrm{mb}$ and to the dashed curve is $\sigma_{e f f}=12 \mathrm{mb}$ (see text). 
This figure "fig1-1.png" is available in "png" format from: http://arxiv.org/ps/hep-ph/9610442v2 


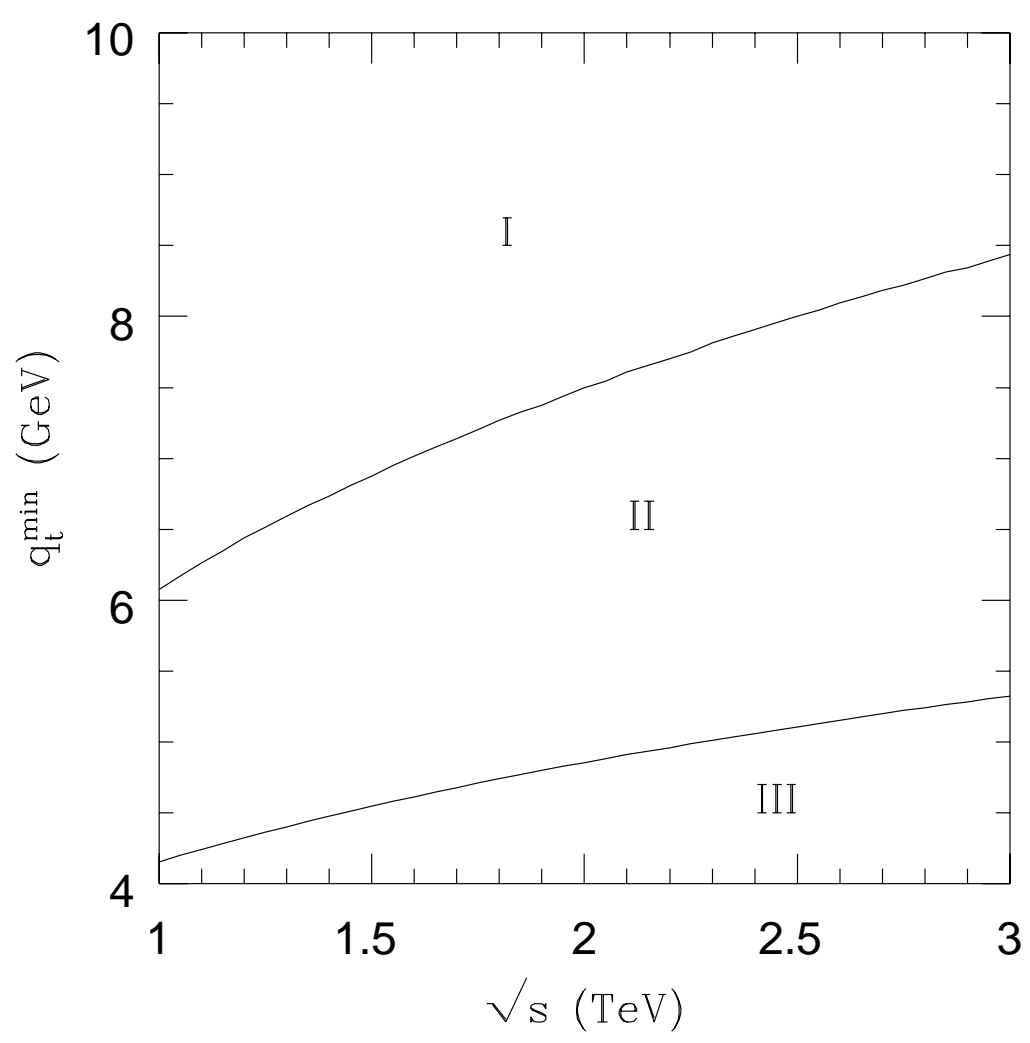




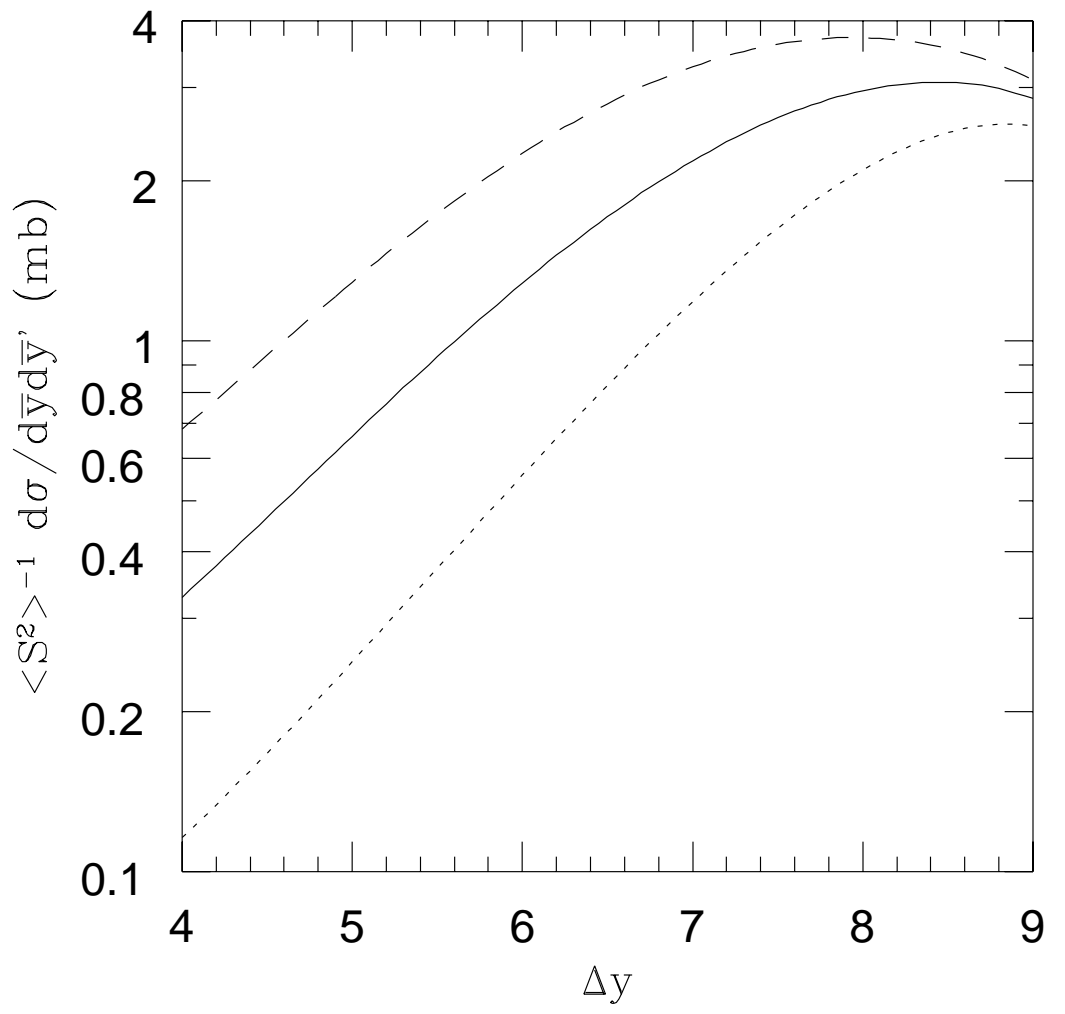

